\title{
ATRIBUTOS FÍSICOS DO SOLO DE UM CAMBISSOLO HÁPLICO EM FLORESTA NATIVA E DE Pinus taeda
}

\author{
Soil physical attributes of haplic cambissol of in native \\ forest and in Pinus taeda manmade forest
}

\author{
André Diogo Dalben ${ }^{1}$, Flora Osaki ${ }^{2}$ \\ ${ }^{1}$ Graduando de Agronomia, PUCPR, Curitiba, PR - Brasil. e-mail: andre_dalben@hotmail.com \\ ${ }^{2}$ Engenheira Agrônoma, Professora Adjunta do Centro de Ciências Agrárias e Ambientais da PUCPR, Curitiba, PR - Brasil. \\ e-mail: rk_osaki@netpar.com.br
}

\begin{abstract}
Resumo
O solo é o principal hábitat que influi no desenvolvimento das plantas, que se relaciona diretamente com o desenvolvimento e crescimento da vegetação. A resistência do solo à penetração é a mais importante, por estar diretamente ligada aos fatores de sua densidade, umidade e granulometria. Este trabalho teve como principal objetivo avaliar as propriedades físicas do solo em áreas de Floresta Nativa (Floresta Ombrófila Mista) e em povoamentos de Pinus taeda. O delineamento utilizado foi o de blocos ao acaso, em arranjo fatorial $2 \times 3$, sendo 6 tratamentos para densidade, umidade e granulometria, com 6 repetições. Os tratamentos estudados foram: fator A - Floresta nativa e povoamentos de Pinus taeda e fator B - três profundidades $0-20 \mathrm{~cm} ; 20-40 \mathrm{~cm} ; 40-60 \mathrm{~cm}$. Para a variável resistência, foram analisados: fator A - Floresta nativa e povoamentos de Pinus taeda e fator B - cinco profundidades $0-5 \mathrm{~cm} ; 5-10$ $\mathrm{cm}$; 10-15 cm, $15-20 \mathrm{~cm}$ e $20-25 \mathrm{~cm}$ com seis repetições. O ensaio foi realizado no município de Tijucas do Sul - PR, entre os meses de maio a outubro de 2006. A resistência à penetração do solo, com exceção da camada de $0-5 \mathrm{~cm}$, foi superior no ecossistema povoamento de Pinus taeda. O teor de umidade no ecossistema Floresta Nativa foi superior ao encontrado no povoamento de Pinus taeda. A densidade do solo foi superior no ecossistema Floresta Nativa nas profundidades $0-20 \mathrm{~cm}$ e $20-40 \mathrm{~cm}$. Em ambos os ecossistemas, a maior densidade do solo foi verificada na profundidade de $40-60 \mathrm{~cm}$. Não houve diferença para teores granulométricos nas diferentes profundidades.
\end{abstract}

Palavras-chave: Solo; Resistência; Densidade; Textura. 


\begin{abstract}
The soil is the main habitat that influences the plant growth, which is interacted to the development of the hole vegetation. The soil resistance to the water penetration is one of the most important environmental factors, because it is strictly linked to density, humidity and soil texture. The main purpose of this research is to evaluate the physical properties of the soil in Subtropical Ombrophilous Forest (Araucaria Forest) and in Pinus taeda manmade forest. The experiment was conducted using a $2 \times 3$ factorial arrangement in random blocks, with 06 treatments for density, humidity and soil texture with 06 replications. The used treatments were: factor A: Native Forest and Pinus taeda manmade forest; factor B: 03 soil deeps: 0-20 cm; 20-40 cm and 40-60 cm. Considering the soil penetrability, it was used the factors $A$ and $B$, with different soil deep: $0-5 \mathrm{~cm} ; 5-10 \mathrm{~cm} ; 10-15 \mathrm{~cm} ; 15-20 \mathrm{~cm}$ and $20-25 \mathrm{~cm}$, with 06 replications. The experiment took place in Tijucas do Sul, Parana State, between May and October of 2006. The resistance to the soil penetrability was greater in the Pinus taeda manmade forest for all soil deeps, except to the 0-5 cm layer. The humidity level in the Native Forest was higher than in manmade forest. The soil density was higher in the Native Forest on the following soil deeps: 0-20 cm and 20-40 $\mathrm{cm}$. In both ecosystems the greatest soil density was observed in 40-60 cm deep. There were no differences between the soil texture in different soil deep.
\end{abstract}

Keywords: Resistance; Soil; Density; Texture.

\title{
INTRODUÇÃO
}

O Brasil é um país com grandes extensões de área, com solo e clima que proporcionam um alto rendimento na produção e boa adaptabilidade de florestas, sejam nativas ou cultivadas. A transformação que o ser humano vem fazendo nos ecossistemas florestais tem alterado drasticamente o equilíbrio da natureza, fazendo com que a dinâmica destes processos seja abalada. Os recursos naturais vêm ultrapassando os limites ecológicos, reduzindo a diversidade biótica e abiótica, que resulta na alteração de inúmeros processos, tanto em florestas naturais, como em cultivadas (FERNANDES; SOUZA, 2003).

Os estudos das modificações em diferentes ecossistemas devem avaliar a estreita relação entre a vegetação e o solo, sendo que primeiramente influencia as propriedades e a dinâmica dos solos, quer diretamente, pelo suprimento de matéria orgânica, ou indiretamente, na estruturação, capacidade de retenção de cátions, aeração, fornecimento de nutrientes, e o comportamento hídrico, que conseqüentemente influencia sobre o tipo de comunidade vegetal local (LONGO et al., 1999).

As alterações de ecossistemas naturais ou cultivados podem promover graves conseqüências à biodiversidade, aos seus solos e, conseqüentemente, à sua sustentabilidade. Dessa forma, estudos que analisam os efeitos causados pela natureza e pela ação antrópica sobre as propriedades do solo constituem importantes recursos para avaliações ou previsões sobre os danos ambientais, servindo como subsídios para discussões sobre a manutenção da qualidade desses solos (LONGO et al., 1999).

Entre as mais importantes propriedades do solo, a resistência à penetração influi na expansão do sistema radicular, com conseqüências sobre a produtividade da floresta, seja natural ou povoamento. A resistência à penetração é o aumento da densidade do solo e o grau aceito depende do propósito a ser atingido (FERNANDES; VITORIA, 1997).

Entre os processos abióticos mais importantes que ocorrem no solo, destaca-se a sua densidade e a sua resistência à penetração, que podem ocorrer naturalmente, resultante do impacto das chuvas, ou pela ação de animais silvestres que transitam nestes locais. Outras fontes de resistência devem-se ao movimento de grandes árvores, resultante da ação do vento, de seu peso ou a pressão exercida pelo crescimento das raízes. As raízes das árvores podem durar por muitas décadas e crescer até tamanhos com efeitos maiores daqueles verificados na agricultura (SEIXAS, 2006). 
A resistência do solo à penetração pode alterar o fluxo de água no solo, reduzir a produtividade do sítio florestal, além de aumentar os níveis de erosão, já que normalmente diminui a taxa de infiltração, aumentando o escorrimento superficial, pois a difusão da água depende do tamanho dos poros (SEIXAS, 2000; SEIXAS; OLIVEIRA; SOUZA, 1998).

Em povoamentos florestais mais densos, verifica-se que a infiltração é bem maior do que naqueles menos densos. A idade da floresta também pode indicar maior ou menor taxa de infiltração da água, sendo maior em florestas mais velhas (ECOLNEWS, 2006).

Outros fatores que podem influir no nível de resistência à penetração envolve a quantidade e distribuição da matéria orgânica (serapilheira, argila), textura e estrutura do solo, porcentagem de umidade do solo, topografia e clima. A presença de uma camada de matéria orgânica (serapilheira) sobre o solo minimiza o impacto da chuva e a lixiviação do solo, protegendo a sua superfície mineral e a fração propriamente dita do solo, argila, silte e areia (SIROIS; STOKES; ASHMORE, 1985).

O manejo dos ecossistemas altera as propriedades físicas e mecânicas no solo, com diferentes níveis de resistência à penetração em função da umidade (KONDO; DIAS JUNIOR, 1999).

Os solos demasiadamente resistentes à penetração impedem a infiltração, tornando-se deficientes em água, além de estarem sujeitos ao aquecimento pronunciado. Por outro lado, solos com baixos teores de água apresentam maior resistência à penetração, enquanto que o aumento da umidade do solo resulta na diminuição da densidade, causando destruição da estrutura do solo, porém, onde a água não se infiltra, ocorre o escorrimento superficial e a perda de nutrientes essenciais à floresta. Assim, a resistência à penetração e umidade do solo bem como os sistemas de plantios devem sempre ser avaliados em conjunto, para o manejo adequado das condições físicas do solo e melhoria da produtividade das florestas, sejam naturais ou cultivadas (SEIXAS, 2000).

O emprego de ações mecânicas exercidas por diferentes máquinas agrícolas para tornar o solo com características físicas desejáveis produz efeitos de ordem mecânica (compressão), que são próprias do solo. Esses podem afetar a circulação de água no solo, promover resistência à penetração em camadas superficiais e subsuperficiais e aumentar o escoamento da água das chuvas (HÉNIN, 1976).

A significativa importância do setor florestal para a economia do país e a escassez de trabalhos sobre resistência à penetração do solo no desenvolvimento florestal justificam a realização do presente trabalho, que visa avaliar as propriedades físicas, bem como verificar o grau de resistência à penetração do solo em dois ecossistemas florestais: cultivado e natural.

\section{MATERIAL E MÉTODOS}

O presente trabalho foi conduzido em campo, no município de Tijucas do Sul, no estado do

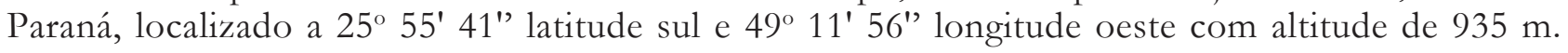

O clima da região é subtropical úmido, mesotérmico, de verões frescos e inverno com geadas freqüentes, sem estação seca, classificado como $\mathrm{Cfb}$. As temperaturas anuais nos meses quentes são de $22^{\circ} \mathrm{C}$ e nos meses frios são inferiores a $18^{\circ} \mathrm{C}$, podendo chegar a $0^{\circ} \mathrm{C}$, a umidade relativa média anual é de $80-85 \%$ e a precipitação média anual é de $1300-1400 \mathrm{~mm}$.

A área experimental encontra-se no complexo fitogeográfico da Floresta Ombrófila Mista (VELOSO; RANGEL FILHO; LIMA, 1991). A área plantada com Pinus taeda é de 30 ha e a de Floresta Ombrófila Mista é de 45 ha. A idade do Pinus taeda é de 21 anos e não foi realizado qualquer tipo de adubação na área, durante seu cultivo.

As variáveis estudadas no presente trabalho foram resistência à penetração do solo $(\mathrm{KPa})$, densidade $\left(\mathrm{g} / \mathrm{cm}^{3}\right)$ e umidade $(\%)$ de solo. A variável resistência à penetração do solo teve como fatores dois ecossistemas - Floresta Nativa e povoamento com Pinus taeda e cinco profundidades - 0-5 cm, 5-10 cm, 10$15 \mathrm{~cm}, 15-20 \mathrm{~cm}$ e $20-25 \mathrm{~cm}$, com um total de dez tratamentos e seis repetições; para as variáveis densidade e umidade do solo teve como fatores: dois ecossistemas - Floresta Nativa e povoamento com Pinus taeda e três profundidades $-0-20 \mathrm{~cm}, 20-40 \mathrm{~cm}$ e $40-60 \mathrm{~cm}$, com um total de seis tratamentos e seis repetições. $\mathrm{O}$ delineamento estatístico experimental aplicado foi o inteiramente casualizado em arranjo fatorial. 
Foi demarcada uma área de $100 \times 100 \mathrm{~m}$ para cada ecossistema $\left(10.000 \mathrm{~m}^{2}\right)$, totalizando 20.000 $\mathrm{m}^{2}$ para os dois ecossistemas, onde foram coletadas amostras aleatoriamente nas três profundidades. Para determinação da resistência à penetração foram realizadas dez medidas e calculada a média para constituir a amostra composta. A resistência do solo foi medida em campo, utilizando-se o penetrômetro marca SOLOTEST. Essa medida foi obtida de $5 \mathrm{em} 5 \mathrm{~cm}$ e de 0 a $60 \mathrm{~cm}$. O princípio da aplicação do penetrômetro consiste na resistência do solo à penetração de uma haste. Esse procedimento é realizado com deslocamento vertical de um bloco de ferro posicionado sobre a haste (CORDEIRO; BATISTA; AMARAL, 1998).

Para a umidade foram realizadas as coletas de solos em dez pontos e o material coletado foi colocado em um recipiente e misturado para compor a amostra composta. O total de amostras para cada ecossistema foi de 18 (seis na profundidade de 0 a $20 \mathrm{~cm}$, seis na profundidade de 20 a $40 \mathrm{~cm}$ e seis na profundidade de 40 a $60 \mathrm{~cm})$.

As amostras foram encaminhadas ao Laboratório de Solos da Pontifícia Universidade Católica do Paraná, São José dos Pinhais - PR, durante o ano de 2006. A determinação da umidade foi realizada da seguinte maneira: pesou-se aproximadamente $5 \mathrm{~g}$ de solo úmido (passado por peneira de 2 $\mathrm{mm}$ de malha) em recipiente de peso conhecido e em balança analítica; submeteu-se à estufa por 24 horas a $105^{\circ} \mathrm{C}$; após esse período, foi esfriado em dessecador e o recipiente foi pesado com o solo seco e calculada a umidade pela fórmula: ( $w_{1}$ : Peso do recipiente $(\mathrm{g}), w_{2}$ : Peso do recipiente + solo $(\mathrm{g})$ e $w_{3}$ : Peso do recipiente + solo seco $(\mathrm{g})$. A fórmula utilizada referente à umidade é expressa em porcentagem (MONTEIRO; FRIGHETTO, 2000).

$$
U=\frac{w_{2}-w_{3}}{w_{3}-w_{1}} \cdot 100
$$

A densidade do solo foi determinada utilizando-se o anel volumétrico. Foram feitas três amostragens por repetição, compondo para os ecossistemas natural e plantado um total de 18 pontos de amostragens cada, totalizando 36 pontos amostrais.

Determinação da densidade $\left(\mathrm{g} / \mathrm{cm}^{3}\right)$ : Após a coleta, as amostras foram colocadas em estufa, onde permaneceram durante 24 horas, sob temperatura de $105^{\circ} \mathrm{C}$. Para o cálculo da densidade do solo, foi utilizada a seguinte expressão: $D S=$ Densidade do solo $\left(\mathrm{g} / \mathrm{cm}^{3}\right), M S=$ Massa do solo seco em estufa a $105^{\circ} \mathrm{C}(\mathrm{g})$ e $V A=$ Volume do anel $\left(\mathrm{cm}^{3}\right)$.

$$
D S=\frac{M S}{V A}
$$

Para verificar a granulometria do solo (\%), as texturas analisadas foram: argila e areia (Método da Pipeta) (TEDESCO et al., 1995).

Os resultados foram submetidos à análise de variância. As variâncias que apresentaram-se homogêneas foram analisadas pelo teste F. Os valores que se mostraram estatisticamente diferentes foram comparados pelo teste de Tukey a $5 \%$ e $1 \%$ de significância.

\section{RESULTADOS E DISCUSSÃO}

Os resultados obtidos mostraram que na profundidade de $0-5 \mathrm{~cm}$, o ecossistema plantado com Pinus taeda apresentou menor resistência à penetração do solo, sendo estatisticamente diferente do ecossistema nativo (TABELA 1). 
TABELA 1 - Resistência à penetração do solo (KPa) em Floresta Nativa e povoamentos com Pinus taeda em cinco profundidades $0-5 \mathrm{~cm}, 5-10 \mathrm{~cm}, 10-15 \mathrm{~cm}, 15-20 \mathrm{~cm}$ e $20-25 \mathrm{~cm}$, Tijucas do Sul, PR

Table 1 - Resistance to soil penetration (Kpa) in Native Forest and in Pinus taeda manmade forest in five different depths 0-5 cm, 5-10 cm, 10-15 cm, 15-20 cm and 20-25 cm, Tijucas do Sul, PR, Brazil

\begin{tabular}{|c|c|c|c|c|c|c|}
\hline & \multicolumn{6}{|c|}{ Profundidade $(\mathrm{cm})$} \\
\hline & $0-5$ & $5-10$ & $10-15$ & $15-20$ & $20-25$ & dms $1 \%$ \\
\hline Nativo & $142,76 \mathrm{Ae}$ & $190,6 \mathrm{Bd}$ & $249,07 \mathrm{Bc}$ & $284,08 \mathrm{Bb}$ & $331,22 \mathrm{Ba}$ & \\
\hline \multirow[t]{3}{*}{ Plantado } & $96,55 \mathrm{Bd}$ & $292,17 \mathrm{Ac}$ & $375,98 \mathrm{Ab}$ & $402,73 \mathrm{Aab}$ & $411,94 \mathrm{Aa}$ & \\
\hline & & & dms $1 \% \quad 16,18$ & & & \\
\hline & & & $\mathrm{CV} \% \quad 5,02$ & & & \\
\hline
\end{tabular}

Médias seguidas por letras distintas (a, b, c, d, e) diferem entre si a 5\% de significância (maiúsculas comparam médias na vertical e minúsculas na horizontal).

De acordo com Camargo e Alleoni (2006), as forças que atuam no solo são resultantes do tráfego de veículos, animais ou pessoas, assim como do crescimento de raízes grandes que empurram as partículas do solo, podendo causar compactação. Assim é possível que o ecossistema plantado, por apresentar um sistema radicular mais homogêneo, possa estar promovendo maior compactação do solo.

Nas profundidades de $5-10 \mathrm{~cm}, 10-15 \mathrm{~cm}, 15-20 \mathrm{~cm}$ e $20-25 \mathrm{~cm}$ o ecossistema de floresta nativa apresentou valores menores e estatisticamente diferentes, quando comparados aos do ecossistema plantado (TABELA 1).

Ao estudar as diferentes profundidades dentro de cada ecossistema, verificou-se que a camada de 0-5 cm apresentou a menor resistência à penetração do solo. É possível observar, na Tabela 1, que ocorreram diferenças estatísticas para todas as profundidades consideradas, tanto para o ecossistema de floresta nativa, quanto para o ecossistema de povoamento com Pinus taeda. Nesse, não foram verificadas diferenças estatísticas entre as profundidades $10-15 \mathrm{~cm}$ e $15-20 \mathrm{~cm}$ e nas profundidades $15-20$ e $20-25 \mathrm{~cm}$. Observou-se, ao longo do perfil do solo, um acréscimo na resistência à medida que aumentou a profundidade.

Em relação à profundidade, as atividades de fatores pedogenéticos resultantes da evolução do solo podem causar o adensamento de camadas subsuperficiais. Os materiais mais finos são transportados da superfície do solo e preenchem os vazios (poros) das camadas inferiores, aumentando sua resistência à penetração (CAMARGO; ALLEONI, 2006).

Martins et al. (2002), estudando quatro povoamentos florestais de Pinus sp, Eucalyptus sp, Hevea brasiliensis e floresta nativa, relataram maior resistência à penetração na profundidade de $0-15 \mathrm{~cm}$ para pínus, eucalipto e hevea e atribuíram esta resposta ao tráfego de máquinas no local. Este resultado difere do obtido para a primeira camada estudada $(0-5 \mathrm{~cm})$, entretanto, deve-se ressaltar que a área plantada com Pinus taeda não esteve sujeita ao trânsito de máquinas. Os mesmos autores relataram que os maiores valores de resistência à penetração foram observados na camada de 20 a $40 \mathrm{~cm}$, semelhantes aos resultados obtidos no presente trabalho.

De acordo com Assis (2006), a fase líquida do solo é composta essencialmente de água e contém minerais dissolvidos e substâncias orgânicas solúveis. A parte líquida ocupa parte ou quase todo o espaço vazio entre as partículas sólidas, de acordo com a umidade do solo. A água do solo pode ser absorvida pelas plantas, drenada para camadas mais profundas ou evaporada, assim salienta-se a importância do conhecimento deste reservatório para as plantas. Além disso, pode promover ou reduzir a atividade biológica do solo (ASSIS, 2006).

Os resultados do teor de umidade para os diferentes tratamentos estão apresentados na Tabela 2. Observa-se que os teores de umidade para os ecossistemas estudados diferem estatisticamente entre si para todas as profundidades testadas (TABELA 2). O ecossistema Floresta Nativa apresentou maior teor de umidade de solo do que o ecossistema povoamento com Pinus taeda.

Dentro do ecossistema Floresta Nativa, as profundidades 0-20 cm, 20-40 cm e 40-60 cm não apresentaram diferenças estatísticas entre si. O mesmo comportamento foi observado para o ecossistema povoamento com Pinus taeda.

Os resultados do teor de umidade do solo (TABELA 2) reforçam os resultados obtidos para a resistência do solo, onde o ecossistema plantado apresentou maior resistência à penetração, indicando que há menor porosidade e, conseqüentemente, menor infiltração e armazenamento de água. 
TABELA 2 - Teor de umidade do solo (\%) em Floresta Nativa e povoamento com Pinus taeda em três profundidades 0-20 cm, 20-40 cm e 40-60 cm, Tijucas do Sul, PR, Brasil

Table 2 - Soil humidity average (\%) in Native Forest and in Pinus taeda manmade forest in three different depths 0-20 cm, 20-40 cm and 40-60 cm, Tijucas do Sul, PR, Brazil

\begin{tabular}{|c|c|c|c|c|}
\hline & \multicolumn{4}{|c|}{ Profundidade $(\mathrm{cm})$} \\
\hline & $0-20$ & $20-40$ & $40-60$ & dms $1 \%$ \\
\hline Nativo & $50,42 \mathrm{Aa}$ & $51,16 \mathrm{Aa}$ & $42,01 \mathrm{Aa}$ & \\
\hline Plantado & $28,61 \mathrm{Ba}$ & $27,16 \mathrm{Ba}$ & $23,55 \mathrm{Ba}$ & \\
\hline \multicolumn{5}{|c|}{$\mathrm{dms} 1 \% \quad 16,15$} \\
\hline \multicolumn{5}{|c|}{$\mathrm{CV} \% \quad 27,37$} \\
\hline
\end{tabular}

Médias seguidas por letras distintas (a, b) diferem entre si a 5\% de significância (maiúsculas comparam médias na vertical e minúsculas na horizontal).

Os resultados para a densidade do solo $\left(\mathrm{g} / \mathrm{cm}^{3}\right)$ são apresentados na Tabela 3. Observa-se que a densidade do solo é maior e estatisticamente diferente para o ecossistema Floresta Nativa, quando comparado ao ecossistema povoamento de Pinus taeda, nas profundidades de $0-20 \mathrm{~cm}$ e $20-40 \mathrm{~cm}$. Costa et al. (2003) analisaram a influência de diferentes tipos de manejo de solo e da cobertura vegetal sobre a densidade do solo e obtiveram valores significativamente diferentes para Latossolo e Gleissolo. $\mathrm{O}$ Gleissolo apresentou valores inferiores de densidade, atribuindo-se o menor valor a sua menor quantidade de matéria orgânica. Na área em estudo, a serapilheira da área plantada com pínus apresentava em média $5 \mathrm{~cm}$ de altura, enquanto que para a floresta nativa atingia apenas $1 \mathrm{~cm}$.

Resultados diferentes aos obtidos neste estudo foram encontrados em trabalho realizado para avaliação das propriedades físicas em Argissolo amarelo sob diferentes coberturas vegetais, como floresta secundária, pastagem e café; foram identificadas maiores densidades do solo para aqueles cultivados com café e pastagem (SÁ; PEREIRA; FONTANA, 2003). Martins et al. (2002) relataram valores de densidade do solo significativamente menor para mata nativa, quando comparado à área plantada com pínus.

Quando consideradas as profundidades dentro do ecossistema Floresta Nativa, verificou-se menor densidade para a camada de $0-20 \mathrm{~cm}$, que diferiu estatisticamente das demais camadas. Resultado semelhante foi verificado por Rigatto, Dedecek e Mattos (2005), que encontraram na profundidade de 0-10 cm menor densidade para todos os sítios estudados (campo e floresta). No solo sob floresta secundária, a menor densidade foi obtida na camada de 0-5 cm por Sá, Pereira e Fontana (2003). No povoamento de Pinus taeda, a menor densidade de solo foi observada para a camada de $0-20 \mathrm{~cm}$, que diferiu estatisticamente da profundidade de $40-60 \mathrm{~cm}$.

TABELA 3 - Densidade do solo dada em $\mathrm{g} / \mathrm{cm}^{3}$ em Floresta Nativa e povoamento com Pinus taeda em três profundidades $0-20 \mathrm{~cm}, 20-40 \mathrm{~cm}$ e $40-60 \mathrm{~cm}$, Tijucas do Sul, PR, Brasil

Table 3 - Soil density shown in $\mathrm{g} / \mathrm{cm}^{3}$ terms in Natural Forest and in Pinus taeda manmade forest in three different depths, 0-20 cm, 20-40 cm and 40-60 cm, Tijucas do Sul, PR, Brazil

\begin{tabular}{lcccc}
\hline & \multicolumn{4}{c}{ Profundidade (cm) } \\
& $\mathbf{0 - 2 0}$ & $\mathbf{2 0 - 4 0}$ & $\mathbf{4 0 - 6 0}$ & $\mathbf{d m s} \mathbf{1 \%}$ \\
\hline Nativo & $2,33 \mathrm{Aa}$ & $2,46 \mathrm{Ab}$ & $2,60 \mathrm{Ab}$ & \\
Plantado & $1,14 \mathrm{Ba}$ & $2,08 \mathrm{Bb}$ & $2,60 \mathrm{Ac}$ & \\
\hline & $\mathrm{dms} \%$ & 0,08 & \\
& $\mathrm{CV} \%$ & 3,29 & \\
\hline
\end{tabular}

Médias seguidas por letras distintas (a, b, c) diferem entre si a 5\% de significância (maiúsculas comparam médias na vertical e minúsculas na horizontal). 
No presente estudo, verificou-se, conforme consta na Tabela 4, que os teores de argila encontrados nos dois níveis de profundidade e nos dois ecossistemas estão acima de $40 \%$, classificando o solo como de textura argilosa. De acordo com a análise estatística, não foram observadas diferenças significativas para os atributos físicos: areia, silte e argila (TABELA 4).

A literatura cita que a classe textural de um solo tem importância primordial como ferramenta auxiliar na determinação da capacidade de suprimento de nutrientes dos sólidos do solo, no fornecimento de água e ar (BRADY, 1974). Entretanto, os tratamentos estudados neste trabalho não apresentam variações significativas quanto a sua classe textural e apresentaram respostas diferentes quanto ao teor de água, resistência à penetração do solo e densidade, sugerindo que tais diferenças estão relacionadas à presença das diferentes coberturas vegetais.

TABELA 4 - Atributos físicos de dois níveis de ecossistemas (nativo e plantado) e dois níveis de profundidade $(0-20 \mathrm{~cm}$ e $20-40 \mathrm{~cm})$ para as variáveis areia, argila e silte dada em porcentagem $(\%)$

Table 4 - Physical attributes in two ecosystems (native and manmade) in two different depths $(0-20 \mathrm{~cm}$ and $20-40 \mathrm{~cm})$ corresponding to sand, clay and silt shown in percentage terms

\begin{tabular}{|c|c|c|c|c|c|c|}
\hline \multicolumn{7}{|c|}{ Profundidade $(\mathrm{cm})$} \\
\hline & \multicolumn{2}{|c|}{ Areia $(\%)$} & \multicolumn{2}{|c|}{ Site $(\%)$} & \multicolumn{2}{|c|}{ Argila (\%) } \\
\hline & $0-20$ & $20-40$ & $0-20$ & $20-40$ & $0-20$ & $20-40$ \\
\hline Floresta Nativa & $36,04 \mathrm{Aa}$ & $36,97 \mathrm{Aa}$ & $9,57 \mathrm{Aa}$ & $18,67 \mathrm{Aa}$ & $44,40 \mathrm{Aa}$ & $42,87 \mathrm{Aa}$ \\
\hline $\begin{array}{l}\text { Floresta Cultivada } \\
\text { (Pinus taeda) }\end{array}$ & $38,67 \mathrm{Aa}$ & $39,65 \mathrm{Aa}$ & $14,40 \mathrm{Aa}$ & $17,47 \mathrm{Aa}$ & $46,35 \mathrm{Aa}$ & $44,35 \mathrm{Aa}$ \\
\hline dms $5 \%$ & \multicolumn{2}{|c|}{9,05} & \multicolumn{2}{|c|}{8,02} & \multicolumn{2}{|c|}{3,59} \\
\hline $\mathrm{CV} \%$ & \multicolumn{2}{|c|}{15,53} & \multicolumn{2}{|c|}{29,72} & \multicolumn{2}{|c|}{7,41} \\
\hline
\end{tabular}

Médias seguidas por letras distintas (a, b, c, d, e) diferem entre si ao nível de significância de 5\% (maiúsculas comparam médias na vertical e minúsculas na horizontal).

\section{CONCLUSÕES}

A resistência à penetração do solo, com exceção da camada de $0-5 \mathrm{~cm}$, foi maior para o ecossistema povoamento com Pinus taeda.

O ecossistema Floresta Nativa apresentou teor de umidade em todas as profundidades superior ao do ecossistema povoamento com Pinus taeda.

A densidade do solo foi superior no ecossistema Floresta Nativa nas profundidades $0-20 \mathrm{~cm}$ e $20-40 \mathrm{~cm}$.

No ecossistema Floresta Nativa, a maior densidade do solo foi verificada na profundidade de 40-60 cm e não diferiu estatisticamente da camada de $20-40 \mathrm{~cm}$.

No ecossistema povoamento com Pinus taeda, a maior densidade do solo foi verificada para a camada de 40-60 cm e diferiu dos demais tratamentos.

A análise granulométrica estabeleceu para todos os tratamentos testados Classe textural argilosa.

\section{AGRADECIMENTOS}

Agradecimentos especiais são creditados ao Dr. Edílson de Oliveira (EMBRAPA), Dr. Daniel Perotto e Dr. Rui Carlos Maranhão Biscaia (IAPAR). 


\section{REFERÊNCIAS}

ASSIS, S. V. de. Umidade do solo. Material didático. Disciplina: Agrometeorologia. Universidade Federal de Pelotas, Faculdade de Meteorologia. Departamento de Meteorologia. Disponível em: <http://www.minerva.ufpel.tche.br/ assis/agro/agro _05.doc>. Acesso em: 28 out. 2006.

BRADY, N. C. The natures and properties of soils. $8^{\text {th }}$ ed. New York: McMillan Publishing Co., Inc, 1974. 647 p.

CAMARGO, O. A.; ALLEONI, L. R. Causas da compactação do solo. Disponível em: < http:/ /www.infobibos.com/Artigos/CompSolo/C3/Comp3.htm>. Acesso em: 28 out. 2006

CORDEIRO, D. G.; BATISTA, E. M.; AMARAL, E. F. Utilização do equipamento penetrômetro de cone para identificação dos níveis de compactação do solo. Instruções Técnicas, n. 15, p. 1-2, dez. 1998.

COSTA, A. M da. et al. Influência da cobertura vegetal na densidade de três solos do cerrado. In: SIMPÓSIO REGIONAL DE GEOGRAFIA: PERSPECTIVAS PARA O CERRADO NO SÉCULO XXI, 2., 2003, Uberlândia. Anais... Uberlândia: [s.n.], 2003. p. 1-8.

ECOLNEWS. A floresta e a infiltração da água. Disponível em: < http//www.ecolnews.com.br/ água/infiltração11.htm>. Acesso em: 16 mar. 2006.

FERNANDES, H. C.; SOUZA, A. P. Compactação de um latossolo-vermelho causada pelo tráfego do "forwarder" Revista Árvores, Viçosa, v. 27, n. 3, p. 279-284, maio/jun. 2003.

FERNANDES, H. C.; VITORIA, E. L. Avaliação dos níveis de compactação de um solo florestal em relação a trafegabilidade das máquinas. Revista Árvore, Viçosa, v. 1, n. 1, p. 521-526, 1997.

HÉNIN, S. Os solos agrícolas. Rio de Janeiro: Florense Universitária; São Paulo: USP, 1976. 334 p. KONDO, M. K.; DIAS JUNIOR, M. S. Compressibilidade de 3 latossolos em função da umidade e uso. Revista Brasileira de Ciência do Solo, Viçosa, v. 23, n. 2, p. 211-218, 1999.

LONGO, R. M.; ESPÍNDOLA, C. R.; RIBEIRO, A. I. Modificações na estabilidade de agregados no solo decorrentes da introdução de pastagens em áreas de cerrado e floresta amazônica. Revista Brasileira de Engenharia Agrícola e Ambiental, Campina Grande, v. 3, n. 3, p. 276-280, 1999.

MARTINS, S. G., et al. Avaliação de atributos físicos de um latossolo vermelho distroférrico sob diferentes povoamentos florestais. CERNE, Lavras, v. 8, n. 1, p. 32-41, 2002.

MONTEIRO, R. T. R.; FRIGHETTO, R. T. S. Determinação da umidade, pH e capacidade de retenção de água no solo. In: FRIGHETTO, R. T. S., VALARINI, P. J. (Coord.). Indicadores Biológicos e Bioquímicos de qualidade do solo. Manual técnico da Embrapa meio ambiente, Jaguariúna, 2000. (Documentos, 21).

RIGATTO, P. A.; DEDECEK, R. A.; MATTOS, J. L. M. de Influência dos atributos do solo sobre a produtividade de Pinus taeda. Revista Árvore, Viçosa, v. 29, n. 5, p. 701-709, set./out. 2005.

SÁ, R. C.; PEREIRA, M. G.; FONTANA, A. Características físicas e químicas de solos de tabuleiros em Sooretama (ES). Floresta e ambiente, Seropédica, v. 10, n. 2, p. 95-99, ago/dez. 2003.

SEIXAS, F.; OLIVEIRA JR.; SOUZA, C. R. Efeito da camada de resíduos florestais na compactação do solo causada pelo transporte primário de madeira. Scientia florestalis, Piracicaba, v. 54, n. 1, p. 9-16, jan. 1998.

SEIXAS, F. Compactação do solo devido a colheita de madeira. 2000. 75 f. Tese (Livre docência) - Escola Superior de Agricultura Luiz de Queiroz, Universidade de São Paulo. Piracicaba, 2000. 
Potencial de danos dos sistemas de colheita de madeira no solo e nas cepas. Disponível em: <http//www.ipef.br/publicações/stecnica/nr30/cap6.pdf>. Acesso em: 10 fev. 2006.

SIROIS, D. L.; STOKES, B. J.; ASHMORE, C. Primary transport of wood on sensitive sites in the Southeast. In: ANNUAL COFE MEETING, 8th., 1985, Proceedings... Corvallis, OR: Council Forest Engineers, 1985. p. 122-127.

TEDESCO, M. J. et al. Análise de solo, planta e outros materiais. 2. ed. Boletim Técnico, Porto Alegre, n. 5, p. 171, 1995.

VELOSO, H. P.; RANGEL FILHO, A. L.; LIMA, J. C. A classificação brasileira, adaptada a um sistema universal. Rio de Janeiro: IBGE, 1991. 123 p. 